\title{
Die Auswirkungen von Angst und Schreck auf Pest und Pestbekämpfung nach zwei Pestschriften des 18. Jahrhunderts
}

Beitrag zum Basler Pest-Kolloquium vom 3. Juni 1978

Von Huldrych M.Koelbing, Urs B. Birchler und Peter Arnold †

Im Frühsommer 1348 fassen zehn junge, vornehme Leute - sieben Damen, drei Herren - eines schönen Morgens den Entschluß, gemeinsam für einige Zeit aus ihrer von der Pest verheerten Vaterstadt auszuziehen. Früh am nächsten Tag lassen sie die fast ausgestorbenen Mauern mit ihren grauenerregenden Visionen des Todes, der drohenden Gesetzlosigkeit und der schmerzlichen Erinnerung an liebe Verstorbene hinter sich und begeben sich auf eines ihrer Landgüter. Sie freuen sich am Jubilieren der Vögel, am frischen Grün der hügeligen Landschaft unter dem weiten Himmel, und unterhalten einander mit amüsanten Geschichten, die die Aufmerksamkeit der Zuhörerinnen und Zuhörer um so mehr fesseln und von den Nöten der Zeit ablenken, als darin das erotische Moment unverhüllt und fröhlich dominiert. - Die zehn jungen Florentiner, mit deren fiktiver Erzählund Fabulierkunst Giovanni Boccaccio ${ }^{1}$ sein «Dekameron» bestreitet, verhielten sich genau so, wie es die Medizin ihrer Zeit und der folgenden Jahrhunderte empfahl: Wer der Pest entgehen wollte und nicht durch amtliche, berufliche oder menschliche Verpflichtungen zurückgehalten wurde, sollte einen verseuchten Ort verlassen, solange er noch gesund war; ferner sollte er sich alle trüben Gedanken aus dem Kopf schlagen. Beide Ratschläge treten in den ärztlichen Pestschriften bis ins 18. Jahrhundert hinein immer wieder auf.

Der gelehrte St. Galler Stadtarzt Vadianus (Joachim von Watt, 1484-1551) beispielsweise riet in seinem 1519 in Basel gedruckten Pestbüchlein, man solle sich in Pestzeiten im kleinen, geschlossenen Kreis zusammenfinden und sich mit Musizieren und fröhlichem Geschichtenerzählen unterhalten ${ }^{2}$. Vadian schrieb übrigens seinen «Kurtz und trüwlich underricht wider die sorglich kranckeyt der Pestilentz ...» für seine Mitbürger nicht etwa in St. Gallen, sondern in Zürich, wohin er selber beim Ausbruch der Epidemie verreist war ${ }^{3}$, einesteils, weil er von einer durchgemachten Erkrankung noch nicht ganz wiederhergestellt war, anderseits um seine Braut Marta Grebel zu heiraten. Seiner Amtspflicht der Stadt St. Gallen gegenüber hatte er Genüge getan, indem er vor der Abreise für die Anstellung eines Apothekers sorgte; Pestbeulen aufzuschneiden und Aderlässe auszuführen war ohnehin nicht die Sache des studierten Mediziners, sondern diejenige des handwerklichen BarbierChirurgen.

Wir möchten nun die medizinischen Auffassungen über die nachteilige Wirkung von Angst und Schreck auf Ausbreitung und Verlauf der Pest aufgrund von zwei einschlägigen Schriften aus dem Besitz des Medizinhistorischen Insti- 
tutes der Universität Zürich erläutern. Es handelt sich um zwei Doktor-Dissertationen aus dem 18. Jahrhundert, die eine 1722 in Halle an der Saale, die andere 1735 in Tübingen vorgelegt und in öffentlicher Disputation verteidigt. Selbstverständlich sind sie auf lateinisch verfaßt. Solche Dissertationen zeichnen sich meistens nicht durch große Originalität und bedeutende neue Erkenntnisse aus; für die beteiligten Doktoranden handelte es sich in der Regel wohl in erster Linie um eine Pflichtübung. Dabei ging es vor allem darum, bei der Disputation eine geltende Lehrmeinung im Sinne des präsidierenden Professors vorzutragen und gegen die nicht immer ernst gemeinten Einwände der Kollegen zu verteidigen. Gerade deswegen können uns solche akademische Alltagspublikationen Aufschluß darüber geben, welche Auffassungen im jeweiligen Zeitpunkt verbreitet, ja vorherrschend waren und das Denken und Handeln einer großen Zahl von Ärzten bestimmten. Nicht die Erleuchtungen der wenigen Großen, sondern das Wissen und Können der vielen Kleinen macht nun aber diejenige Medizin aus, die die Patienten und das Publikum zu spüren bekommen.

Die Doktordisputation (disputatio pro gradu doctoris) wurde oben als Pflichtübung bezeichnet. Die beiden hier behandelten Disputationstexte - derjenige von Christian Sigismund Finger in Halle (1722) noch ausgesprochener als derjenige von Christian Ludwig Moegling in Tübingen (1735) - zeigen aber, daß manche der jungen Verfasser sich für ihr Thema begeisterten und es mit viel Ernst, Sorgfalt und Bemühen um richtige Einsichten und Schlußfolgerungen behandelten. Wieviel eigene, originale Gedanken sie auch im Rahmen einer konventionellen Darstellung des gegebenen Problems entwickelt haben, das könnte oft nur ein umständlicher Vergleich mit anderen Schriften ähnlicher Thematik zeigen, und auch dann bliebe es zunächst offen, wie weit die Urheberschaft solcher Gedanken beim Doktoranden und wie weit sie bei seinem Promotor lag ${ }^{4}$.

Man kann fragen, ob es sich bei unseren beiden Dissertationen nur der Form nach um akademische Erörterungen handelt - hatte nicht auch das Thema der Pest selbst seine aktuelle praktische Bedeutung verloren? Es ist höchst unwahrscheinlich, daß Finger oder Moegling während ihrer Studienzeit die Pest aus eigener Anschauung kennengelernt haben, und auch für die beiden Praesides, die Professoren Alexander Camerarius (1695-1736) in Tübingen und Georg Daniel Coschwitz (1679-1729) in Halle, ist es fraglich.

Moegling bezeichnet 1635 als das letzte Pestjahr in Tübingen ${ }^{5}$. Zwischen 1708 und 1712 trat die Seuche noch in den deutschen Hafen- und Handelsstädten Bremen, Hamburg, Danzig und Königsberg auf, ebenso in Prag; im weiteren Verlauf des Jahrhunderts machte sie sich fast nur noch in Polen, Rußland und der Türkei bemerkbar (1737 nochmals in Danzig) ${ }^{6}$. 
Aber 1720 war die Pest noch einmal mit aller Wucht in Marseille ausgebrochen und hatte sich über die umliegende Provence verbreitet (1720-1722). Diese unerwartete Epidemie schreckte die Ärzte und die Ratsherren in ganz Westund Mitteleuropa auf: Das Unglück, das Marseille, Aix und Avignon heimsuchte, konnte eines Tages auch über Wien, Leipzig oder Zürich wieder hereinbrechen. Man mußte nach wie vor über die Krankheit Bescheid wissen, um ihr gegebenenfalls begegnen zu können; man mußte die Abwehr und Eindämmung der Seuche planen und organisieren, ehe diese heranrückte. Die Pest von Marseille war ein Warnsignal, welches sozusagen das ganze 18.Jahrhundert hindurch wirksam blieb $^{7}$, den Behörden beim Ausbau des öffentlichen Gesundheitswesens immer vor Augen stand und die Ärzte zum Nachdenken über ihre Möglichkeiten und Pflichten in Epidemiezeiten anregte. Dabei schien es unseren Vorfahren geboten, auch Furcht und Schreck als reale Elemente eines Pestausbruches aufzufassen und ernst zu nehmen. Da die individuelle Widerstandskraft gegen die Krankheit durch Gemütsbewegungen vermindert werden konnte, mußten auch die Behörden sich fragen, ob nicht ihre kollektiven Schutzmaßnahmen die Bevölkerung unnötig und verhängnisvoll ängstigen und dadurch schwer schädigen könnten. Ausführlich und systematisch untersucht Finger diese Fragen, mehr kursorisch und anekdotisch tut es Moegling.

Christian Sigismund Finger ${ }^{8}$ geht von einem Sprichwort aus, das besagt, schlimmer als die Pest sei die Angst vor der Pest. Gewichtige Autoren bezeugen, so fährt er fort, daß Leute die Pest bekamen, einzig weil ihnen der Lärm eines vorbeifahrenden Leichenwagens einen Schreck einjagte. Die Möglichkeit, daß Krankheiten durch Angst oder Schreck ausgelöst werden können, bezweifelt Finger grundsätzlich nicht: «Täglich erleben die Praktiker, daß Pocken und Masern bei vielen Patienten durch die Furcht ausgelöst werden. Da wird einer krank vom Anblick eines anderen, der an Pocken oder Masern leidet ... ${ }^{9}$ (In Wirklichkeit ist nach unserem heutigen Wissen auch hier Ansteckung, VirusÜbertragung im Spiel.) - Für die Pest hält Finger freilich derartige Berichte nicht für zuverlässig: Ohne das Hinzutreten des ansteckenden Pestgiftes (contagium pestilens) vermögen Furcht und Schreck keine Pest hervorzurufen ${ }^{10}$. Aber diese beiden Gemütsbewegungen verschlimmern die Krankheit, und die Angst macht schon den gesunden Menschen anfälliger für die Ansteckung. Wie das vor sich geht, ist Gegenstand ausführlicher Erörterungen.

Wir halten uns nicht bei Fingers Ausführungen über das Wesen der Krankheit auf. Die Pest ist jedenfalls «eine epidemische, ansteckende, höchst akute und für die meisten tödliche Krankheit». ${ }^{11} \mathrm{Zu}$ ihrem Wesen gehört Fieber, auch wenn man dieses nicht immer feststellen kann. Die Heilung ist das Werk der «natura», 
die als eine den Organismus regierende, seine innere Tätigkeit («Bewegungen» motus) steuernde Kraft verstanden wird. Sie treibt das Krankheitsgift (miasma pestilentiale, virus morbosum), das sich mit dem von ihm befallenen Teil der Körpersäfte zu Krankheitsstoff (materia peccans) vereinigt, mittels des Herzschlages aus den edleren, inneren Organen hinaus unter die Haut. Dort wird diese Materie abgesondert und ausgeschieden: durch die unmerkliche Ausdünstung und den Schweiß, aber auch in die Pestkarbunkel, Pestbeulen und punktförmigen Hautblutungen. (Solche Petechien, maculae petechiales, treten indessen vor allem beim Fleckfieber auf, das davon seinen Namen hat.) Der ruhige und gesetzmäßige Ablauf dieser Absonderungs- und Ausscheidungsprozesse setzt zweierlei voraus: erstens, daß die Natur die entsprechenden Bewegungen prompt und kräftig einleitet und sie sodann ausdauernd und gleichmäßig in Gang hält, bis zum guten Ende; zweitens, daß die Wege der Ausscheidung, d.h. die Blutgefäße und die Poren des Unterhautgewebes und der Haut, das richtige Maß an Durchgängigkeit haben. Die Kraft der Bewegung wie vor allem die Durchgängigkeit der Wege hängt von der Spannung, vom Tonus ab, der im Körper und seinen Teilen herrscht. Dieser darf natürlich nicht zu gering, aber auch nicht zu groß sein: eine übermäßige Spannung (strictura) der Fasern in den oberflächlichen Geweben verschließt die Poren und verunmöglicht so der auszuscheidenden Krankheitsmaterie den Ein- und Durchtritt, so daß sie ins Innere des Körpers zurückfließen muß und dort die lebenswichtigen Organe zerstört.

In diesen natürlichen Heilungsprozeß durch Absonderung und Ausscheidung greifen nun Furcht und Schreck verhängnisvoll ein ${ }^{12}$. Die Furcht (timor) macht nicht nur den Geist, sondern auch die Natur in ihrem unbewußten Wirken unsicher und verhindert dadurch die Austreibung der Krankheitsmaterie; der Schreck (terror) steigert die Spannung der Fasern der Haut und des Unterhautgewebes und blockiert dadurch die Ausscheidungswege.

Finger hält, wie uns scheint, die Furcht für gefährlicher als den Schreck; da aber aus einem großen Schrecken häufig eine tiefsitzende, dauernde Furcht entsteht, bewertet er die beiden Affekte schlußendlich als gleich schlimm. Während der reine Schreck jedoch nur den bereits im Gang befindlichen Absonderungsund Ausscheidungsprozeß unterbrechen kann, macht die Angst die Natur zu jeder Zeit unfähig zur Abwehr: selbst wer noch gesund ist, verliert dadurch schon seine Widerstandskraft gegen das Pestgift und fällt somit der Infektion und der Krankheit leichter zum Opfer.

Wir haben es hier mit einer psychosomatischen Pestlehre von spezifisch hallescher Prägung zu tun. Die Gemütsbewegungen wirken gleichzeitig und gleichsinnig im seelisch-geistigen 
wie im körperlichen Bereich, letzteres über die "natura». Als zielstrebige, autonome und unbewußte Regentin des Körpers stimmt diese mit der «anima» Georg Ernst Stahls (1660-1734) überein. Stahl war von der Universitätsgründung 1694 an bis 1716 Medizinprofessor in Halle gewesen, und Fingers Lehrer Coschwitz gehörte zu seinen Anhängern. In Stahls «anima » oder «natura - er benützte beide Ausdrücke - lebt die aristotelische Vorstellung von der Seele als Lebensprinzip des Organismus wieder auf. Für ihr Wirken im Körper bedient sie sich des Tonus, und zwar im dynamischen Sinne («tonische Bewegung») wie im statischen (Spannung der Fasern). Die Wichtigkeit des Tonus für den regelrechten Ablauf der Lebensprozesse, die Finger hervorhebt, entspricht aber auch der Auffassung Friedrich Hoffmanns (1660-1742), der zweiten Koryphäe der jungen medizinischen Fakultät von Halle.

Aufgrund der hier skizzierten Deutung des Krankheitsgeschehens unterzieht Finger am Schluß seiner Dissertation ${ }^{13}$ die zur Eindämmung einer Pestepidemie üblichen Maßnahmen einer kritischen Besprechung. Mit den Handels- und Reisesperren gegen infizierte Orte, die die Einschleppung der Pest verhüten sollen, erklärt er sich einverstanden, «sofern nur dafür gesorgt wird, daß niemand die zum Leben nötigen und zur Heilung wichtigen Dinge entbehren muß und daß die erlassenen Gesetze die Grenzen der Nächstenliebe und Barmherzigkeit nicht verletzen ». ${ }^{14}$ Er würde also Flüchtlinge aus Pestgebieten wohl nicht gnadenlos am Stadttor zurückweisen.

Ist die Pest jedoch in einer Stadt ausgebrochen, dann hält es Finger mit Richard Mead ${ }^{15}$ (1673-1754) in London für die oberste Pflicht der Behörden, ihre Mitbürger von der Notwendigkeit und Uneigennützigkeit aller ihrer Anordnungen zu überzeugen, angefangen mit der Pflicht zur Meldung der Erkrankten an die Behörden. Alles muß als Hilfe, nichts darf als Strafe erscheinen ${ }^{16}$. Die einzelnen Maßnahmen, auf die Finger nun eingeht, haben alle den Zweck, die Kranken möglichst gut von den Gesunden zu trennen und von diesen das ansteckende Krankheitsgift fernzuhalten. Finger will «prüfen, ob sie wirklich der guten Absicht entsprechen und ob sie nicht in Tat und Wahrheit zur Ausbreitung der Pest beitragen und ihr Wüten verschlimmern ». ${ }^{17}$

Im Prinzip ist die Absonderung der Pestkranken notwendig und nützlich; aber Finger ist nicht der Meinung, daß zu diesem Zweck nun etwa jeder Patient ins Pestspital gebracht werden müßte. Denn «für gewöhnlich sind die Pestspitäler (nosocomia pestilentialia) so beschaffen, daß sie - ohne oder selbst gegen die Absicht der Behörden - vor stinkendem Schmutz starren. Rücksichtslos werden die Kranken zusammengepfercht, wie es gerade kommt. Das Pflegepersonal entstammt dem leichten Volk und besorgt die Kranken nicht mit Liebe, sondern nur um des Geldes willen. Die große Zahl der Patienten verunmöglicht es den Ärzten, Chirurgen und Pflegern, jedem einzelnen die gebotene Sorgfalt zuzuwenden. Es 
fehlt selbst an Bettwäsche; eine Beachtung der einzelnen Symptome und eine danach sich richtende Behandlung findet nicht statt. Die diesen Krankenhäusern vorstehenden Pest-Ärzte und -Chirurgen sind im allgemeinen waghalsig, wenig erfahren und von geringem Ansehen; sie ziehen ihre Erfahrung aus dem Tod anderer. Niemand, glaube ich, wird es uns zur Last legen, wenn wir davon abraten, alle an Pest Erkrankten ohne Unterschied in die öffentlichen Krankenhäuser zu überführen. ${ }^{18}$

Unter diesen Umständen kann die Überführung ins Krankenhaus ein verhängnisvolles seelisches Trauma (wie man heute sagt) sein. Finger zitiert Augustus Quirinus Rivinus (Bachmann, 1652-1723), der berichtet, wie 1680 in Leipzig manche Patienten auf dem Transport ins Pestspital vor lauter Schreck und Angst gestorben seien ${ }^{19}$. (Rivinus gehörte zu den nicht ganz seltenen Ärzten, die überzeugt waren, daß Furcht und Schreck den Menschen überhaupt erst empfänglich für die Krankheit machen ${ }^{20}$.) Nach Finger haben die Pestkranken eine höhere Chance, mit dem Leben davonzukommen, wenn sie unbesorgt und ruhig in ihrem eigenen Haus bleiben können, jedoch in einem besonderen Zimmer abgesondert und dort liebevoll gepflegt werden. Er bezweifelt nicht, daß sich genügend vertrauenswürdige Leute für den Pflegedienst finden lassen, sofern sie nur die Gewißheit haben, daß man sie ebenfalls im Hause behält und nicht ins Spital abschiebt, falls sie selber angesteckt werden ${ }^{21}$.

Ins Pestspital gebracht werden soll also nur, wer daheim nicht gepflegt werden kann. Finger schlägt deshalb vor, von Staates wegen die Einwohner in drei Klassen einzuteilen: eine erste Klasse der wohlhabenden Bürger, die überhaupt keine staatliche Hilfe brauchen; eine zweite der mäßig Bemittelten, die ihre Kranken zwar zu Hause absondern und pflegen können, dazu jedoch staatliche Unterstützung - Geld, Medikamente, Pflegepersonal - brauchen; schließlich eine dritte der ganz Armen, die niemand und nichts haben und für deren Pflege nur das Pestspital in Frage kommt ${ }^{22}$. Da sie sonst vollkommen hilflos und verloren wären, setzt unser Autor bei ihnen auch keine so große Angst vor jenem wenig einladenden Ort voraus.

Dieser handfeste Vorschlag einer Klasseneinteilung der gesamten Stadtbevölkerung mit seiner Kritik an den Notspitälern und deren ärztlicher Leitung dürfte unseres Erachtens eher von Coschwitz stammen, der als Professor eine Respektsperson war, als von seinem jungen Doktoranden Finger.

Eine weitere Absonderungsmaßnahme bestand im Verschließen infizierter Häuser, wobei die gesunden Hausbewohner zusammen mit den Kranken eingesperrt wurden. Manchmal ließ sich auf diese Weise die Pest tatsächlich eindämmen, wie das von Burri nach I. Wehrli aus Baden im Aargau mitgeteilte Beispiel von 1665 
zeigt $^{23}$. Finger hält es für schrecklich und verkehrt, Menschen auf diese Weise einzukerkern: die seelisch bedingte Schwächung der Kranken wie der Gesunden überwiegt den allfälligen Nutzen dieser rigorosen Maßnahme. Unser Autor ist offensichtlich froh, bei Mead Zahlen gefunden zu haben, die diese Behauptung stützen ${ }^{24}$.

An den infizierten Häusern wurden mancherorts Warnzeichen angebracht. Finger hält das für klug - nur sollen die Kranken im Innern des Hauses nichts davon merken! Beifällig erwähnt er den Vorschlag Ysbrand van Diemerbroecks, auch gesunde Personen, die aus einem Pesthaus kommen, sollten ein entsprechendes Zeichen auf sich tragen, damit man ihnen ausweichen könne ${ }^{25}$ - eine Anregung, die offensichtlich von der mittelalterlichen Kennzeichnung der Aussätzigen inspiriert ist, unseres Wissens aber nie verwirklicht wurde.

Sorgen bereitete den Verantwortlichen ferner der Hausrat, mit dem Pestkranke gelebt hatten. Sollte man einfach alles verbrennen, am besten mitsamt dem Haus? Das Niederbrennen eines Hauses, selbst wenn man das Übergreifen des Brandes auf die Nachbarhäuser verhindern kann, ist sicher ein Angst und Schreck erregendes Ereignis und sollte nach Finger schon deshalb unterbleiben. Er kann zudem auf französische und britische Erfahrungen hinweisen, die die Nutzlosigkeit solch radikalen Durchgreifens beweisen. Auch von den Gebrauchsgegenständen verbrenne man nur, was man nicht vollkommen vom Pestgift reinwaschen kann: Wolle, Seide, Pelze, Kissen u. dgl. ${ }^{26}$

Recht eingehend befaßt sich Finger mit den Arzten. $1656 \mathrm{kam}$ in Rom die Schutzkleidung mit Schnabelmaske, Hut und Talar auf, die man von vielen Abbildungen her kennt. «Ein teuflischer Anblick», kommentiert Finger, der Gesunde und Kranke zutiefst erschrecken und den Krankheitsverlauf nur verschlimmern kann ${ }^{27}$. Demgegenüber fällt der bescheidene Nutzen, den eine solche Gewandung dem Arzt bieten kann, nicht ins Gewicht. Für ihn liegt der beste Schutz in seiner Charakterstärke und Seelenruhe. Ein ängstlicher Arzt, dem es vor dem Anblick (und dem Geruch) der Kranken graust, wird sehr leicht angesteckt und von der Krankheit dahingerafft, aber zahlreich waren und sind die Ärzte, die bei Pest- und anderen Epidemien «auf Gott vertrauend, ohne Anwendung von Gegengiften (alexipharmaca) unbeirrt den Weg ihrer Pflicht gehen und nie von einer solchen Krankheit ergriffen werden». Gottvertrauen und Pflichtbewußtsein ist das beste Antidot gegen das Pestgift. Erfahrene und erprobte Ärzte, die sich in Epidemiezeiten weigern, ihre Berufspflicht zu erfüllen, machen sich an den Verheerungen der Seuche mitschuldig ${ }^{28}$. - Die Flucht des Arztes vor der Pest gilt im 18. Jahrhundert nicht mehr als standeswürdig.

Christian Sigismund Finger und sein Lehrer Georg Daniel Coschwitz rechnen 
also mit der Verbreitung der Pest durch Übertragung des Pestgiftes von Mensch zu Mensch, durch Gebrauchsgegenstände und durch die Luft. Sie bejahen grundsätzlich die Maßnahmen, die diese Übertragung verhindern oder doch erschweren können, aber sie haben für sie nur relativen Wert. Die Ruhe des Gemütes und die Zuversicht unter den gesunden wie den kranken Gliedern des Gemeinwesens soweit möglich zu erhalten, scheint ihnen ebenso wichtig, um eine Epidemie zu überstehen; was die Menschen schreckt und ängstigt, ist keine gute Pestbekämpfung. Ist diese Haltung nun weise oder schwächlich?

Nach unserem heutigen Verständnis konnten die mittelalterlich-frühneuzeitlichen Absonderungsmaßnahmen infizierte, aber noch nicht schwer erkrankte Menschen hindern, ihre Pestbazillen weiterzutragen, und sie konnten die direkte Ansteckung durch Tröpfcheninfektion (bei der Lungenpest) sowie die allfällige zwischenmenschliche Übertragung durch Flöhe ${ }^{29}$ verhüten. Sie waren jedoch völlig unwirksam gegen die Verschleppung der Erreger durch Ratten, von deren Rolle für die Pestverbreitung man noch nichts wußte. Die auf der Ansteckungshypothese beruhenden Vorkehrungen waren somit die einzig möglichen, aber sie waren tatsächlich nur von relativem Wert. Wenn Coschwitz, Finger und andere sie so auffaßten, können wir sie kaum tadeln.

Zudem haben seuchenpolizeiliche und andere präventivmedizinische Maßnahmen immer ihre psychologische Seite. Sofern man nicht die davon Betroffenen unter ständiger Kontrolle hat wie in einem Gefängnis oder einer Kaserne und selbst da gibt es ja Lücken in der Überwachung -, lassen sie sich nur dann wirksam durchführen, wenn die Menschen sie verstehen und deshalb für sich selber bejahen. Das war früher schon so (wie z. B. die Studie von Hanspeter Ruesch über die Zürcher Pestabwehr zeigt ${ }^{30}$ ) - und es wird in Zukunft so sein, bei uns wie in den Ländern der Dritten Welt. Mit einer von Schreck geschüttelten und von Angst erfüllten Bevölkerung läßt sich nur schwer eine vernünftige Krankheitsbekämpfung treiben; darin hatten Finger, Coschwitz und ihre mehr oder weniger illustren Gesinnungsgenossen gewiß recht, auch wenn sie offensichtlich allzu genau zu wissen glaubten, auf welchen Wegen Furcht und Schreck den Widerstand des Organismus gegen die Pest zu lähmen vermögen.

Im Bereich der psychologischen Pestabwehr, die jenen Ärzten so wichtig war, kann man natürlich sehr leicht auf Irrwege geraten. Christian Ludwig Moegling nennt aufgrund zahlreicher von ihm zitierter Autoritäten den Schreck neben der schlechten Luft und dem Ansteckungsstoff als den dritten Kausalfaktor für das Zustandekommen der Pest ${ }^{31}$ und wirft dann jene Frage auf, die - wie er sagt eine besondere "politisch-medizinische Erörterung» verdienen würde: Wäre es nicht besser, die Existenz der Seuche zunächst zu verheimlichen, bis die ersten 
Vorkehrungen dagegen getroffen sind, da doch die öffentliche Bekanntmachung eines Pestausbruches einen Schock für alle bedeutet? Aus dieser Besorgnis heraus habe der Rat der Stadt Danzig im Jahre 1709, als die ersten kleinen Pestherde zu glimmen begannen, kurzerhand ein Verbot erlassen, von außergewöhnlichen, bedrohlichen Krankheiten zu reden ${ }^{32}$. Wir haben Mühe, den psychologischen Nutzen eines solchen Erlasses einzusehen.

Sicher ist andererseits, daß im Sommer 1720 die Pest in Marseille nur deswegen durch den Orientfahrer «Grand St-Antoine» eingeschleppt werden und nachher sich ausbreiten konnte, weil die Quarantäne nachlässig gehandhabt, ja umgangen wurde und man die Natur der ersten Todesfälle, die auf dem Schiff und in der Stadt vorkamen, vertuschte ${ }^{33}$. Nicht nur Schonung und Takt haben ihre Berechtigung; oft kann nur der Mut zur harten Wahrheit ein Unheil abwehren.

\section{Anmerkungen}

${ }^{1}$ Boccaccio, Giovanni, Il decamerone. Prima giornata, la pesta di Firenze - introduzione.

2 Milt, Bernhard, Vadian als Arzt, St. Gallen 1959, S. 90.

${ }^{3}$ A.a. O., S. 45.

${ }^{4} \mathrm{Zu}$ vergleichen wäre namentlich die Pestschrift von Fingers Promotor Georg Daniel Coschwitz, Kurtzer und wohlgemeinter Bericht wie bey jetzigen an vielen Orthen sich hervorthuenden ansteckenden Krankheiten und sogenandter Pest ein jeglicher Hauß-Vater mit denen seinigen sich praeserviren könne, Halle 1725, 16 S. (Zit. nach Biraben - s. Anm. 6 Bd. 2, S. 240.) Wir haben bisher kein Exemplar dieser Schrift finden können.

${ }^{5}$ Moegling, Christian Ludwig, Disputatio inauguralis medica de peste, quam ... praeside D. Alexandro Camerario ... pro gradu doctoris publico eruditorum examini submittit Christianus Ludovicus Moegling Tubingensis, Tübingen 1735, 28 S., 102 §§. Deutsche Übersetzung von Peter Arnold und Huldrych M.Koelbing, bisher nicht publiziert. - §35.

${ }^{6}$ Biraben, Jean-Noël, Les hommes et la peste en France et dans les pays européens et méditerranéens, Paris und den Haag 1975, Bd. 1, «Annexe IV».

${ }^{7}$ Ackerknecht, Erwin H., Geschichte und Geographie der wichtigsten Krankheiten, Stuttgart 1963, S. 15. - Kocher, Alexander, Die Pestepidemie zu Marseille 1720-1722; ihre Bedeutung für das medizinische Denken, Diss. med., Zürich 1967.

${ }^{8}$ Finger, Christian Sigismund, Dissertatione inaugurali medica timoris et terroris in peste noxam ... praeside Dn. Georgio Daniel Coschwitz ... pro gradu doctoris ... eruditorum examini subjiciet Christianus Sigismundus Finger, Halle an der Saale 1722, 48 S., 34 §§. Deutsche Übersetzung in: Christian Sigismund Fingers Dissertation Über den schädlichen Einfluß von Furcht und Schreck bei der Pest (Halle 1722), von Huldrych M.Koelbing unter Mitarbeit von Urs B.Birchler, Aarau 1979 (Veröffentlichungen der Schweizerischen Gesellschaft für Geschichte der Medizin und der Naturwissenschaften 33).

${ }^{9}$ A.a. O., Prooemium - Vorwort.

10 A.a.O., § 16.

11 A.a.O., § 2 . 
12 A.a. O., §§ 20-25.

13 A.a. O., §§ $26-34$.

14 A.a. O., § 26 .

${ }^{15}$ Mead, Richard, A short discourse concerning pestilential contagion, and the methods to be used to prevent it, London 1720. Lateinische Übersetzung London 1721.

${ }^{16}$ Finger, a.a.O. (s. Anm. 8), § 26.

17 A.a. O., § 27.

18 A.a. O., § 28 .

19 A.a. O., § 29.

${ }^{20}$ Finger, a. a. O., § 26. - Moegling, a.a.O. (s. Anm. 5), § 67. - Biraben (s. Anm. 6), Bd. 2, S. $37 f$., führt die Auffassung, daß die Angst vor der Pest diese herbeiziehe, auf Avicenna (980-1037) zurück.

${ }^{21}$ Finger, a. a. O. (s. Anm. 8), § 29.

${ }^{22}$ A. a. O., § 28.

${ }^{23}$ Burri, Hans Rudolf, Das Aufhören der Pest in der Schweiz, gegenwärtiger Stand der Forschung, Vortrag 14. Juni 1975 in Zürich, vervielfältigtes Ms., S. 9. - Wehrli, Ida, Das öffentliche Medizinalwesen der Stadt Baden im Aargau ... 1349-1798, Aarau [1927], S. $114 \mathrm{f}$.

${ }^{24}$ Finger, a. a. O. (s. Anm. 8), § 30.

25 A.a.O., § 31 .

26 A.a. O., § 33 .

27 A.a. O., § 32 .

28 A.a. O., § 31 .

${ }^{29}$ Die Theorie, daß neben der Infektkette Ratte-Floh-Mensch die Kette Mensch-FlohMensch bei den europäischen Pestepidemien eine wichtige Rolle gespielt haben kann, wird heute mit besonderem Nachdruck von Biraben (s. Anm. 6) vertreten. Biraben kann sich dabei u.a. auf Georg Sticker und vor allem auf Ernst Rodenwaldt stützen: Sticker, Georg, Die Pest, 2. Teil: Die Pest als Seuche und als Plage, Gießen 1910. - Rodenwaldt, Ernst, Pest in Venedig 1575-1577, Heidelberg 1953. Vgl. Pollitzer, Robert, La peste, Genf 1954, S. 401-404 über den Menschenfloh Pulex irritans als möglichen Pestüberträger.

${ }^{30}$ Ruesch, Hanspeter, Das "Erlufftungshaus» in Weiach, 1720-1721, Beitrag zu einer Studie der obrigkeitlichen Pestbekämpfung im alten Zürich, Vortrag 3.Juni 1978 in Basel.

${ }^{31}$ Moegling, a.a.O. (s. Anm. 5), §§ 67-71.

32 A.a. O., § 75 .

${ }^{33}$ Biraben, a.a. O. (s. Anm. 6), Bd. 1, S. $230 \mathrm{ff}$. 


\section{Summary}

Fear and fright in plague - 18th century views

The unexpected outbreak of plague, in 1720, at Marseilles alerted public authorities as well as physicians all over Europe. The nature of plague and the usefulness of the various measures supposed to prevent or to check an epidemic were discussed in medical writings throughout the 18 th century. It was generally admitted that fear and fright might greatly diminish the resistance to infection. In a doctoreal thesis defended before the Medical Faculty of Halle an der Saale in 1722, Christian Sigismund Finger explains the psychosomatic processes involved in terms of the pathophysiological concepts prevailing then in Halle: fear and fright (a) impair the actions of the individual's Nature, the guiding principle of the body (G. E. Stahl's anima, actually borrowed from Aristotle), and they (b) block the evacuation of morbid matter towards the skin by enhancing the tonus of the subcutaneous fibers (here we see F.Hoffmann's influence). In his MD thesis of 1735, Christian Ludwig Moegling of Tübingen lists fright as the third causal factor of plague, besides corruption of the air and contagion.

Such ideas could lead to practical consequences. Finger (a pupil of G.D. Coschwitz) reviews the administrative and sanitary measures that used to be taken in plague epidemics in the light of their possible frightening or reassuring effects. Following R.Mead, he deprecates the forced inclusion of whole families in their houses. He gives an appalling description of plague hospitals (not founded, however, in personal observation); these institutions, he thinks, are just well enough for the poorest. He insists upon separation of the sick from the healthy (preferably in their own homes) and upon cleaning or destruction of possibly infected clothes and furniture. Like Mead, Finger relies more on the citizens' insight and responsibility than on authoriarian severity. Moegling, on the other hand, thinks that the spreading of frightening rumours might be prohibited by decree.

Prof.Dr.med.Huldrych M. Koelbing

Medizinhistorisches Institut

der Universität Zürich

Rämistraße 71

8006 Zürich

Mein Doktorand Peter Arnold ist am 7. Oktober 1978 im Alter von 32 Jahren gestorben. Trotz schwerster Behinderung durch Krankheit hat er 1976 das medizinische Staatsexamen absolviert und sich anschließend mit Freude an die Übersetzung und Bearbeitung von Moeglings Pestschrift (s. Anm. 5) gemacht. Die Früchte seiner Arbeit sollen der Wissenschaft noch zugänglich gemacht werden.

H.M. K. 